\title{
Photothermal excitation setup for a modified commercial atomic force microscope
}

\author{
Holger Adam, ${ }^{1}$ Sebastian Rode,,${ }^{1}$ a) Martin Schreiber, ${ }^{1}$ Kei Kobayashi, ${ }^{2}$ Hirofumi Yamada, ${ }^{2}$ \\ and Angelika Kühnle ${ }^{1, b)}$ \\ ${ }^{1}$ Institute of Physical Chemistry, Johannes Gutenberg University Mainz, Duesbergweg 10-14, \\ 55099 Mainz, Germany \\ ${ }^{2}$ Department of Electronic Science and Engineering, Kyoto University, Katsura, Nishikyo, \\ Kyoto 615-8510, Japan
}

(Received 2 December 2013; accepted 20 January 2014; published online 7 February 2014)

\begin{abstract}
High-resolution imaging in liquids using frequency modulation atomic force microscopy is known to suffer from additional peaks in the resonance spectrum that are unrelated to the cantilever resonance. These unwanted peaks are caused by acoustic modes of the liquid and the setup arising from the indirect oscillation excitation by a piezoelectric transducer. Photothermal excitation has been identified as a suitable method for exciting the cantilever in a direct manner. Here, we present a simple design for implementing photothermal excitation in a modified Multimode scan head from Bruker. Our approach is based on adding a few components only to keep the modifications as simple as possible and to maintain the low noise level of the original setup with a typical deflection noise density of about $15 \mathrm{fm} / \sqrt{\mathrm{Hz}}$ measured in aqueous solution. The success of the modification is illustrated by a comparison of the resonance spectra obtained with piezoelectric and photothermal excitation. The performance of the systems is demonstrated by presenting high-resolution images on bare calcite in liquid as well as organic adsorbates (Alizarin Red S) on calcite with simultaneous atomic resolution of the underlying calcite substrate. (C) 2014 AIP Publishing LLC. [http://dx.doi.org/10.1063/1.4864084]
\end{abstract}

\section{INTRODUCTION}

Atomic force microscopy (AFM) is widely used as a most powerful method for direct surface imaging. In the last decades, the high-resolution imaging capability of frequency modulation (FM) AFM has been demonstrated not only under well-controlled ultra-high vacuum conditions, ${ }^{1}$ but also in more application-relevant environments such as ambient or liquids. $^{2}$ Modifications in the AFM setup ${ }^{3,4}$ have opened up the possibility for atomic-resolution imaging of single-crystal surfaces $^{2,5,6}$ as well as investigating organic adsorbates with unmatched resolution. ${ }^{7,8}$ Recently, the high sensitivity of FM AFM in liquids has enabled the three-dimensional visualization of hydration layers ${ }^{9}$ as well as mapping the interfacial layered structure of other liquids. ${ }^{10-12}$

In liquids, however, the widely used method for exciting the cantilever oscillation by a piezoelectric transducer results in a so-called "forest of peaks"13 in the resonance spectrum, which originates from acoustic modes of the liquid and the setup. These additional peaks in the excitation spectrum are known to cause serious problems in identifying the resonance of the cantilever itself. A major obstacle arises from the fact that the resonance frequencies of the acoustic modes are subject to frequent changes, e.g., when the water evaporates during the scan. This causes the peaks to move, which disturbs or even aborts a measurement when the peaks drift into the locking range of the detection system. Moreover, quantitative data interpretation, especially with respect to dissipative forces, is

a) Present address: SmarAct GmbH, Schuette-Lanz-Str. 9, 26135 Oldenburg, Germany.

b) kuehnle@uni-mainz.de difficult. This is due to the fact that a significant, but generally unknown percentage of the excitation energy is lost for exciting acoustic waves in the system, which, in turn, drive the cantilever indirectly. ${ }^{14}$

A most elegant method for overcoming these obstacles is driving the cantilever directly. Several techniques have been explored for direct cantilever excitation, including magnetic modulation ${ }^{15,16}$ and thermal excitation ${ }^{17}$ using resistive heating. However, magnetic modulation requires magnetic cantilever tips and resistive heating can easily result in unwanted temperature changes in the sample volume with the risk of inducing large thermal drift. As an alternative strategy, a modulated laser beam has been used for photothermal cantilever excitation. ${ }^{18,19}$ For this method, an additional laser is focused at the cantilever base. To excite the cantilever oscillation, the power of the laser beam is modulated at the actual resonance frequency. This photothermal excitation method has been successfully implemented in a few (home-built) AFM scan heads optimized for low-noise applications. ${ }^{20-22}$

Here, we present a simple and easy-to-implement design for a widely used scan head, namely, a modified scan head of a Multimode AFM from Bruker. The scan head used in this work has been optimized for operation in liquids before to arrive at a low deflection noise density of about $15 \mathrm{fm} / \sqrt{\mathrm{Hz}} .4$ The modification requires the addition of an external excitation laser, which is power-modulated at the actual resonance frequency of the cantilever. The success of this modification is illustrated by presenting the comparison of resonance spectra obtained with piezoelectric and photothermal excitation. To demonstrate the high performance of the modified system, we present high-resolution images taken on bare calcite(10.4) 


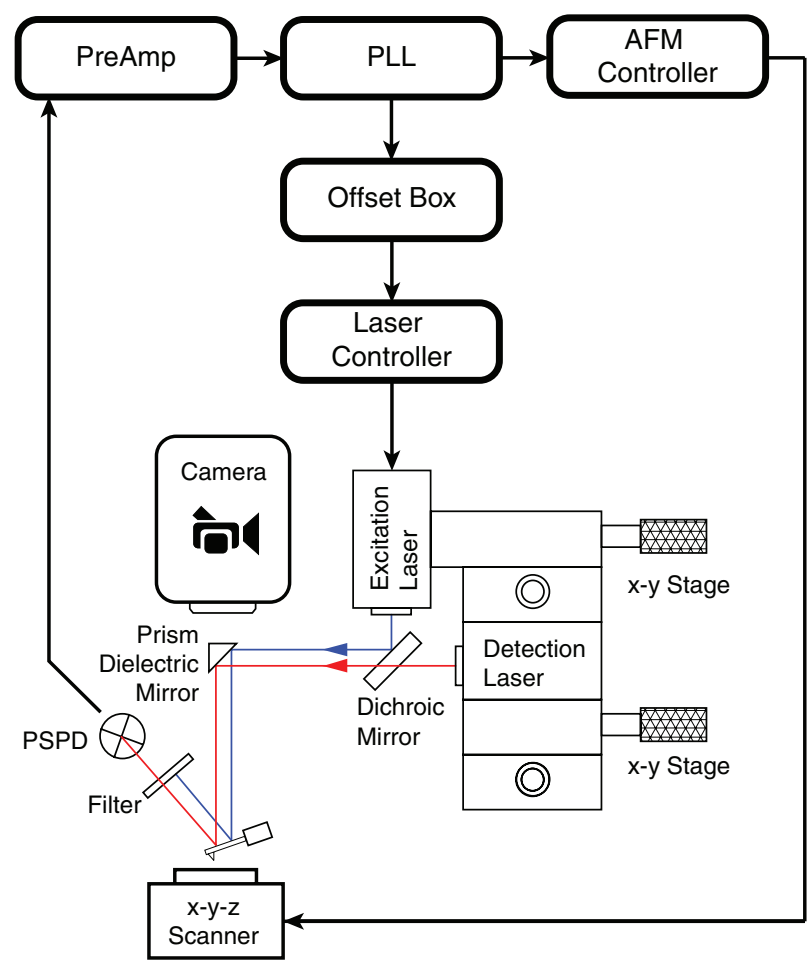

FIG. 1. Components of the modified scan head, including the detection and the exciting laser beams. In the electronics, an offset box and a laser controller box are added into the feedback loop.

as well as Alizarin Red S (ARS) on calcite(10.4) in aqueous solution using the FM AFM imaging mode.

\section{IMPLEMENTATION OF PHOTOTHERMAL EXCITATION}

\section{A. Optical pathway}

For implementing photothermal excitation in our modified setup, ${ }^{4}$ an excitation laser diode is added to the system (Fig. 1). The excitation laser diode (PhoxX 405-120, $405 \mathrm{~nm}$ wavelength, $120 \mathrm{~mW}$ maximum output power) from Omicron-Laserage $\mathrm{GmbH}$ is coupled into the system using a single-mode optical fiber (LDM.FASY.APC, Omicron-Laserage $\mathrm{GmbH}$ ), a fiber collimator (60FC-4-M2033 , Schäfter+Kirchhoff $\mathrm{GmbH}$ ), and micro-focus optics (5MS88-33-S, Schäfter+Kirchhoff $\mathrm{GmbH}$ ). A double-axes micrometer stage (XYPG25-A, Misumi) is used for positioning the laser spot at the base of the cantilever beam. The laser beam is directed towards the cantilever by using a dichroic mirror (DMLP567, Thorlabs) and a right angle prism dielectric mirror (MRA05-E02, Thorlabs). In front of the positionsensitive photodetector (PSPD), we add a filter (type E62-977, Edmund Optics) to avoid irradiation of the PSPD by the excitation laser. A photograph of the modified scan head is shown in Fig. 2.

\section{B. Electronics}

Photothermal excitation requires the modulation of the excitation laser power at the actual resonance frequency of

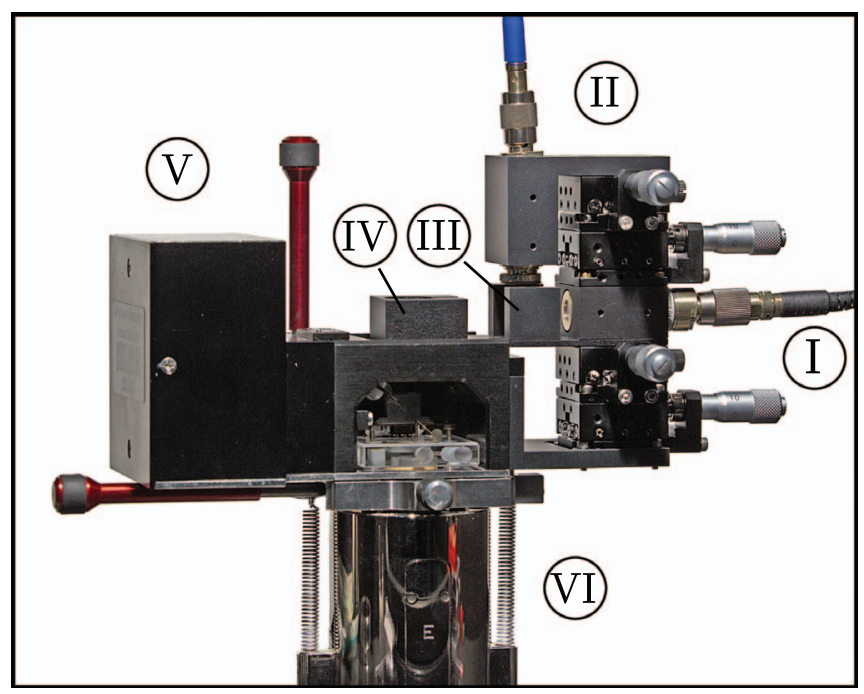

FIG. 2. Photograph of the modified scan head. The components are: I - detection laser, II - exciting laser, III - dichroic mirror, IV - prism dielectric mirror, V - preamplifier, VI - Multimode scanner.

the cantilever. To implement this, the electronics of the feedback loop are modified. The oscillation signal from the PSPD is passed to an amplifier (home-built preamplifier and band pass filter), which is designed in a way to allow for optimum noise reduction while at the same time keeping the bandwidth large enough for measurement. ${ }^{4}$ From there, the signal is fed into a phase-locked loop (PLL, easyPLL plus Detector, and Controller from Nanosurf AG). The easyPLL plus Detector determines the actual resonance frequency. The easy PLL plus Controller generates an excitation signal, which is based on a reference oscillation signal at the current resonance frequency multiplied by a factor that is determined by the amplitude feedback loop to keep the oscillation amplitude constant, which is chosen by the operator through the amplitude setpoint. The excitation signal is passed onto a home-built offset box, which is used to add an adjustable offset. This box adjusts the range of the input signal for the laser controller, as the modulation input of the used laser diode is limited to $1 \mathrm{~V}$ against ground. The offset is added using a low-noise, low-drift summing amplifier based on an operational amplifier (OP213 from Analog Devices, Inc.) and a low-noise voltage reference (ADR441 from Analog Devices, Inc.). For each measurement, we chose the smallest offset that is sufficient to adjust the modulation input. The laser modulation is implemented using the laser controller from the excitation laser diode. The modulation input voltage is used to set the laser light intensity. An internal feedback loop with an additional photodiode adjusts the laser light intensity with a bandwidth of $3 \mathrm{MHz}$ in a way that the intensity is proportional to the actual modulation voltage input. Additionally, the diode is temperature-stabilized.

\section{RESULTS}

\section{A. Excitation spectra}

To demonstrate the success of our modifications, we present a comparison of the resonance spectrum using the 

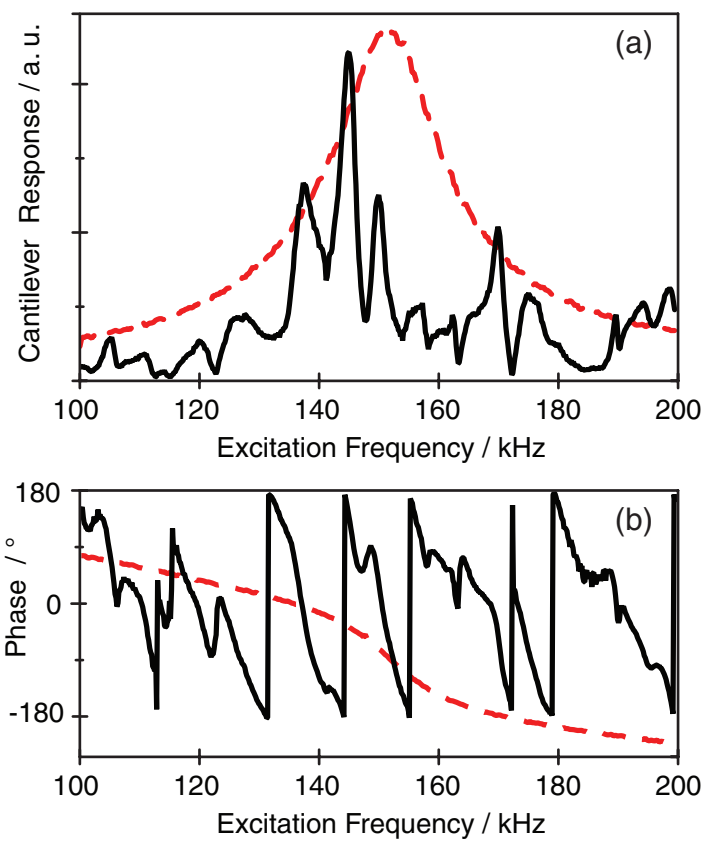

FIG. 3. (a) Resonance spectra obtained with a HF2 lock-in amplifier (Zurich Instruments) using piezoelectric (solid curves) and photothermal excitation (dashed curves). A "forest of peaks" is revealed for piezoelectric excitation, while only one well-defined peak is obtained for photothermal excitation. (b) Corresponding phase response, showing multiple phase shifts for piezoelectric excitation, while only one phase shift is associated with the single resonance peak for photothermal excitation. An overall linear decrease of the phase in the given frequency range can be explained by the filter used in the preamplifier. Data are taken using a NCHAuD cantilever from NanoSensors with a sensitivity of $12 \mathrm{~nm} / \mathrm{V}$ and a quality factor of around 9 .

commercial piezoelectric transducer and photothermal excitation (Fig. 3) taken in supersaturated calcium carbonate solution. The spectra are taken several micrometer away from the calcite(10.4) sample, to exclude an influence of the sample on the spectra. We used a NCHAuD cantilever from NanoSensors with a resonance frequency of $152 \mathrm{kHz}$ and a quality factor of around 9. In this experiment, we measured a deflection noise density of $12 \mathrm{fm} / \sqrt{\mathrm{Hz}}$ and a sensitivity of $12 \mathrm{~nm} / \mathrm{V}$. The photothermal spectrum was acquired with an excitation laser intensity that was adjusted to obtain approximately the same resonance peak height as revealed before for the acoustically excited spectrum. As can be seen, several peaks are revealed when driving the cantilever with the piezoelectric transducer. In contrast, only one well-defined resonance peak is obtained in the case of photothermal excitation. This shape greatly simplifies the data acquisition, as can be understood by considering the corresponding phase shift, which is the relevant signal for the PLL to determine the actual resonance frequency. A well-defined resonance peak is associated with a single, steep $90^{\circ}$ pass in the phase at the corresponding resonance frequency. In contrast, several peaks in the resonance spectrum result in a superposition of the corresponding shifts in the phase. If the additional peaks are stationary, imaging might be possible, but usually with reduced sensitivity. However, the frequencies of the acoustic modes are usually drifting in time. In this case, they can cause the PLL to lock out. Reliable data acquisition is impossible in this case.

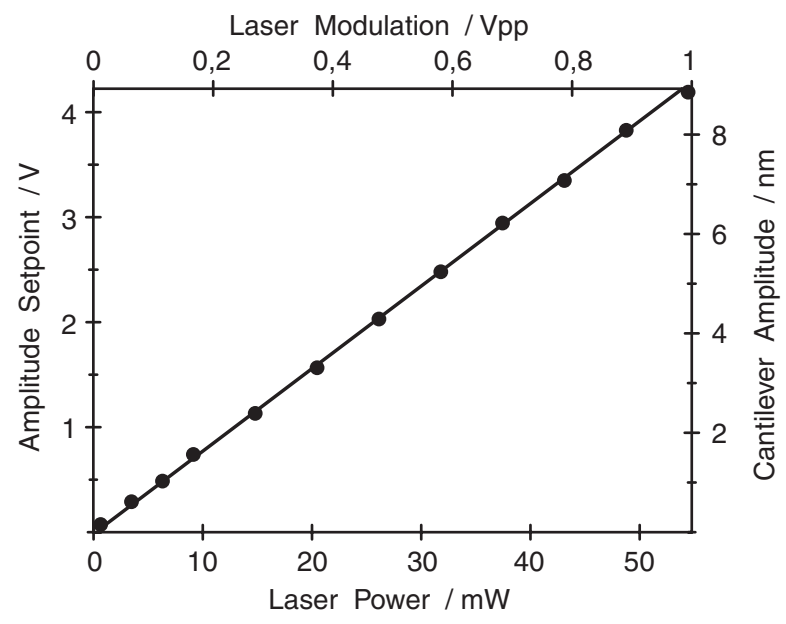

FIG. 4. Dependence of the cantilever oscillation amplitude on the modulation voltage input. The data were taken with a NCHAuD cantilever from NanoSensors with a sensitivity of approximately $14 \mathrm{~nm} / \mathrm{V}$ and a quality factor of about 9 . The laser power corresponding to a given modulation input was measured using a DC signal at the laser controller input.

\section{B. Instrumental parameters}

Routinely obtained noise levels for the modified setup measured with a NCHAuD cantilever from NanoSensors in aqueous solution correspond to a spectral noise density of about $15 \mathrm{fm} / \sqrt{\mathrm{Hz}}$ at a sensitivity of approximately $15 \mathrm{~nm} / \mathrm{V}$. Under optimum conditions, noise levels as low as $6 \mathrm{fm} / \sqrt{\mathrm{Hz}}$ are obtained. Depending on the modulation voltage input of the laser controller, the physical oscillation amplitude of the cantilever is changed. Fig. 4 shows the dependence of the cantilever oscillation amplitude on the modulation voltage input of the laser controller box to verify that a linear relationship is obtained. The smallest amplitude that still allows for stable operation is about $0.5 \mathrm{~nm}$ which is obtained with a modulation voltage of $70 \mathrm{mV}_{\mathrm{pp}}$. Amplitudes as large as $10 \mathrm{~nm}$ can be achieved with the maximum modulation voltage of $1 \mathrm{~V}_{\mathrm{pp}}$. This can be of interest when considering an instrumental setup that allows for a flexible switch from tapping mode to FM imaging, which is often of interest for a first identification of interesting areas and subsequent high-resolution imaging.

\section{AFM imaging}

The performance of the modified instruments is demonstrated by presenting high-resolution images taken in FM mode with photothermal excitation. Calcite crystals are purchased from Korth Kristalle (Kiel). For each experiment, a freshly cleaved calcite sample is placed in a closed liquid cell (MTFML tapping mode fluid cell, Bruker). A droplet of supersaturated calcium carbonate solution is then introduced into the cell. Atomically resolved images of the natural cleavage plane of calcite, namely, calcite(10.4) can be taken on a routine basis. In Fig. 5(a), a representative image of the calcite(10.4) surface is shown, taken with a NCHAuD cantilever from NanoSensors with a resonance frequency of $147 \mathrm{kHz}$ and a quality factor of about 8 . The oscillation amplitude was $0.6 \mathrm{~nm}$. We measured a sensitivity of $14 \mathrm{~nm} / \mathrm{V}$ and a deflection noise density of $11 \mathrm{fm} / \sqrt{\mathrm{Hz}}$. An atomic-size defect can 

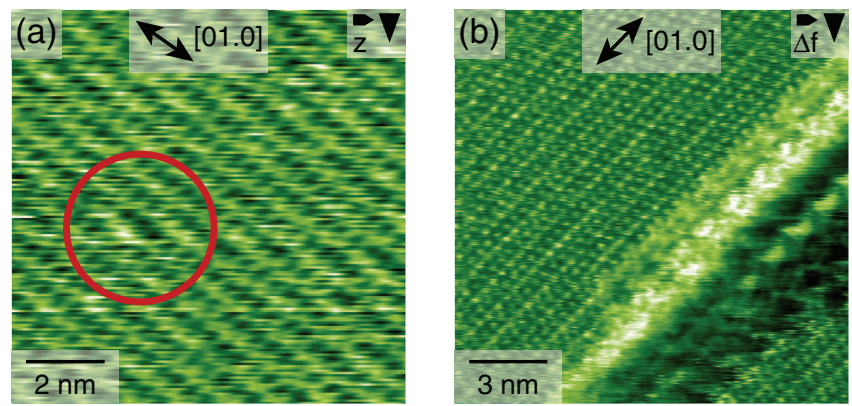

FIG. 5. (a) Representative FM AFM image of the bare calcite(10.4) surface taken in supersaturated calcium carbonate solution. An atomic-size defect is seen (circle). The image was taken with a NCHAuD cantilever from NanoSensors with a resonance frequency of $147 \mathrm{kHz}$. The image was taken with scan speed of 12 lines per second. (b) High-resolution FM AFM image after deposition of Alizarin Red S (ARS) molecules. ${ }^{7}$ Needle-like adsorbates are observed. Note that atomic resolution of the underlying substrate is obtained simultaneously. The image was taken with an ACTG cantilever from AppNano with a resonance frequency of $165 \mathrm{kHz}$ and a quality factor of 9 . The image was taken with scan speed of 12 lines per second.

be identified in the image (marked by a circle), confirming true atomic-resolution imaging.

To further illustrate the high-resolution capability, we perform high-resolution images after deposition of an organic adsorbate, namely, Alizarin Red S (3,4-dihydroxy-2anthraquinonesulfonic acid sodium salt). ${ }^{7}$ For this experiment, an ACTG cantilever from AppNano with a resonance frequency of $165 \mathrm{kHz}$ and a quality factor of about 9 was used. The oscillation amplitude was $0.5 \mathrm{~nm}$. We measured a sensitivity of $17 \mathrm{~nm} / \mathrm{V}$ and a deflection noise density of $16 \mathrm{fm} / \sqrt{\mathrm{Hz}}$. As can be seen in Fig. 5(b), a characteristic, needle-like structure is clearly resolved with inner resolution. Most importantly, we simultaneously achieve atomic resolution of the underlying substrate, which confirms the high resolution capability even in the presence of protruding species.

\section{CONCLUSION}

We present a detailed description for modifying a commercial AFM scan head (Multimode from Bruker) to implement photothermal excitation. A simple design is chosen to maintain the ease of use of the original instrument. The suc- cess of the modification is demonstrated by comparing excitation spectra taken with piezoelectric and photothermal excitation, clearly showing the reduction of the "forest of peaks" to a single, well-defined resonance curve. Atomically resolved AFM images taken in FM mode in aqueous solution on calcite(10.4) with and without organic adsorbates are presented to demonstrate the performance of the modified system.

\section{ACKNOWLEDGMENTS}

We are grateful to Ralf Bechstein for most stimulating discussions.

${ }^{1}$ F. J. Giessibl, Rev. Mod. Phys. 75, 949 (2003).

${ }^{2}$ T. Fukuma, K. Kobayashi, K. Matsushige, and H. Yamada, Appl. Phys. Lett. 87, 034101 (2005).

${ }^{3}$ T. Fukuma, M. Kimura, K. Kobayashi, K. Matsushige, and H. Yamada, Rev. Sci. Instrum. 76, 053704 (2005).

${ }^{4}$ S. Rode, R. Stark, J. Lübbe, L. Tröger, J. Schütte, K. Umeda, K. Kobayashi, H. Yamada, and A. Kühnle, Rev. Sci. Instrum. 82, 073703 (2011).

${ }^{5}$ S. Rode, N. Oyabu, K. Kobayashi, H. Yamada, and A. Kühnle, Langmuir 25, 2850 (2009).

${ }^{6}$ S. Rode, R. Hölscher, S. Sanna, S. Klassen, K. Kobayashi, H. Yamada, W. G. Schmidt, and A. Kühnle, Phys. Rev. B 86, 075468 (2012).

${ }^{7}$ M. Schreiber, M. Eckardt, S. Klassen, H. Adam, M. Nalbach, L. Greifenstein, F. Kling, M. Kittelmann, R. Bechstein, and A. Kühnle, Soft Matter 9, 7145 (2013).

${ }^{8}$ T. Sugihara, I. Hayashi, H. Onishi, K. Kimura, and A. Tamura, Chem. Phys. 419, 74 (2013).

${ }^{9}$ T. Fukuma, Y. Ueda, S. Yoshioka, and H. Asakawa, Phys. Rev. Lett. 104, 016101 (2010).

${ }^{10}$ T. Hiasa, K. Kimura, and H. Onishi, J. Phys. Chem. C 116, 26475 (2012).

${ }^{11}$ T. Hiasa, K. Kimura, and H. Onishi, Colloids Surf. A 396, 203 (2012).

${ }^{12}$ T. Hiasa, K. Kimura, and H. Onishi, J. Phys. Chem. C 117, 5730 (2013).

${ }^{13}$ T. E. Schäffer, J. P. Cleveland, F. Ohnesorge, D. A. Walters, and P. K. Hansma, J. Appl. Phys. 80, 3622 (1996).

${ }^{14}$ D. Kiracofe and A. Raman, Nanotechnology 22, 485502 (2011).

${ }^{15}$ S. P. Jarvis, A. Oral, T. P. Weihs, and J. B. Pethica, Rev. Sci. Instrum. 64, 3515 (1993).

${ }^{16}$ W. H. Han, S. M. Lindsay, and T. W. Jing, Appl. Phys. Lett. 69, 4111 (1996).

${ }^{17}$ A. C. Hillier and A. J. Bard, Rev. Sci. Instrum. 68, 2082 (1997).

${ }^{18}$ N. Umeda, S. Ishizaki, and H. Uwai, J. Vac. Sci. Technol. B 9, 1318 (1991).

${ }^{19}$ G. C. Ratcliff, D. A. Erie, and R. Superfine, Appl. Phys. Lett. 72, 1911 (1998).

${ }^{20}$ T. Fukuma, Rev. Sci. Instrum. 80, 023707 (2009).

${ }^{21}$ A. Labuda, K. Kobayashi, D. Kiracofe, K. Suzuki, P. H. Grütter, and H. Yamada, AIP Adv. 1, 022136 (2011).

${ }^{22}$ A. Labuda, K. Kobayashi, Y. Miyahara, and P. Grütter, Rev. Sci. Instrum. 83, 053703 (2012). 